\title{
Formalism for testing theories of gravity using lensing by compact objects. III: Braneworld gravity
}

\author{
Charles R. Keeton \\ Department of Physics \& Astronomy, Rutgers University, \\ 136 Frelinghuysen Road, Piscataway, NJ 08854; \\ keeton@physics.rutgers.edu \\ A. O. Petters \\ Departments of Mathematics and Physics, Duke University, \\ Science Drive, \\ Durham, NC 27708-0320; \\ petters@math.duke.edu
}

Accepted in Phys Rev D

\begin{abstract}
Braneworld gravity is a model that endows physical space with an extra dimension. In the type II Randall-Sundrum braneworld gravity model, the extra dimension modifies the spacetime geometry around black holes, and changes predictions for the formation and survival of primordial black holes. We develop a comprehensive analytical formalism for far-field black hole lensing in this model, using invariant quantities to compute all the geometric optics lensing observables: bending angle, image position, magnification, centroid, and time delay. We then make the first analysis of wave optics in braneworld lensing, working in the semi-classical limit. Through quantitative examples we show that wave optics offers the only realistic way to observe braneworld effects in black hole lensing. We point out that if primordial braneworld black holes exist, have mass $M_{\bullet}$, and contribute a fraction $f_{\mathrm{bh}}$ of the dark matter, then roughly $\sim 3 \times 10^{5} \times f_{\mathrm{bh}}\left(M_{\bullet} / 10^{-18} M_{\odot}\right)^{-1}$ of them lie within our Solar System. These objects, which we call "attolenses," would produce interference fringes in the energy spectra of gamma-ray bursts at energies $E \sim 100\left(M_{\bullet} / 10^{-18} M_{\odot}\right)^{-1} \mathrm{MeV}$ (which will soon be accessible with the GLAST satellite). Primordial braneworld black holes spread throughout the universe could produce similar interference effects. If they contribute a fraction $\Omega_{\bullet}$ of the total energy density, the probability that gamma-ray bursts are "attolensed" is at least $\sim 0.1 \Omega_{\bullet}$. If observed, attolensing interference fringes would yield a simple upper limit on $M_{\bullet}$. Detection of a primordial black hole with $M_{\bullet} \lesssim 10^{-19} M_{\odot}$ would challenge general relativity and favor the braneworld model. Further work on lensing tests of braneworld gravity must proceed into the physical optics regime, which awaits a description of the full spacetime geometry around braneworld black holes.
\end{abstract}

Keywords: gravitational lensing, gravity theories, extra dimension

\section{INTRODUCTION}

Gravitational lensing has emerged as a powerful and far-reaching tool in astrophysics and cosmology [1, 2, 3]. In this series we are showing how lensing can also be employed to test theories of gravity. Papers I [4] and II 5] developed an analytical formalism for identifying the lensing signatures of gravity models that fall within the post-post-Newtonian (PPN) framework, even probing out to third order in such models. These studies uncovered some surprising universal relations among lensing observables that helped us make specific predictions that are testable with current or near-future instrumentation.

In this paper we examine a gravity model that lies outside the PPN framework, namely, type II RandallSundrum braneworld gravity 6]. According to this model, familiar 4-dimensional spacetime is actually a submanifold (a "brane") in a 5-dimensional spacetime (the "bulk"), with the extra dimension characterized by a curvature radius $\ell$ which could be as large as $\sim 0.2 \mathrm{~mm}$ [7]. One intriguing prediction of the model is that braneworld black holes might be produced at energies as low as $\sim 1$
$\mathrm{TeV}$, which could lead to observable Hawking radiation in the forthcoming Large Hadron Collider [8] or create specific signatures in cosmic ray showers [9].

Another important prediction is that braneworld black holes produced in the early universe might survive to the present day. Primordial black holes are predicted to have formed from density fluctuations in the very early universe, with a mass spectrum that increases rapidly towards low masses 10, 11. In general relativity, black holes smaller than $\sim 10^{-19} M_{\odot}$ would have evaporated by now through Hawking radiation [12]. Compared with their GR counterparts, however, braneworld black holes evaporate more slowly [13] and may have accreted more efficiently in the early universe [14, 15]. Together, these effects may allow primordial braneworld black holes as small as $M_{\bullet} \sim 1 \mathrm{~kg} \sim 10^{-30} M_{\odot}$ to survive to today [15, 16]. The implication is that primordial braneworld black holes may contribute some fraction of the unknown dark matter. Gravitational lensing offers a crucial test of this hypothesis if we can identify appropriate lensing scenarios that carry a clear imprint of braneworld gravity. That is our goal. 
An exact metric for the spacetime geometry induced by a braneworld black hole is still unknown. In the far-field or weak-deflection regime, it is well established that braneworld black holes are described by the GarrigaTanaka metric 17, 18, 19]. In the near-field or strongdeflection regime, various metrics are believed to approximate the true spacetime geometry (e.g., [20]). These different metrics have been used to explore gravitational lensing by braneworld black holes (see the review by Majumdar \& Mukherjee [16]). For example, Kar \& Sinha 21] computed the light bending angle for the GarrigaTanaka and tidal Reissner-Nordström metrics. Majumdar \& Mukherjee [22] determined the light bending angle, image position, and magnification for the weakdeflection regime of the Myers-Perry metric. Eiroa 23] and Whisker 24] studied the bending angle, image position, and magnification for the strong-deflection regime of the Myers-Perry and tidal Reissner-Nordström metrics, respectively. Still other metrics may prove useful for studying the approximate lensing properties of braneworld black holes (cf. 20, 24]).

We present a thorough study of lensing in braneworld gravity, including a realistic assessment of prospects for observing braneworld effects in astrophysical lensing scenarios. We focus on weak-deflection lensing for two reasons. First, the images that appear in the strongdeflection limit carry important near-horizon effects but are exceedingly difficult to observe [25, 26]. Second, we shall argue that wave optics will play a crucial role in lensing tests of braneworld gravity, and wave optics observables are dominated by the two images that appear in the weak-deflection regime. Since the Garriga-Tanaka metric correctly describes the spacetime geometry in the far-field regime of a braneworld black hole, we develop the full analytical infrastructure for weak-deflection lensing in this metric.

We rederive the light bending angle in the GarrigaTanaka metric, but express it for the first time in terms of invariant quantities (Section प). We go beyond the bending angle and use invariant quantities to compute the observable properties of the lensed images: positions, magnifications, and time delays (Sections IIIIV). We then consider for the first time wave optics effects in braneworld black hole lensing (Section $\nabla$ ). Finally, we examine a variety of applications of braneworld lensing. We show that traditional astrophysical lensing scenarios will be unable to measure braneworld effects in the foreseeable future (Section VI). However, an application of wave optics that we call "attolensing" provides exciting opportunities for observing braneworld effects (Section (VII). In particular, we point out that if primordial braneworld black holes exist and contribute to the dark matter, they must exist not only throughout the universe but also within our Solar System. Attolensing will provide a crucial test of this prediction of the braneworld model.

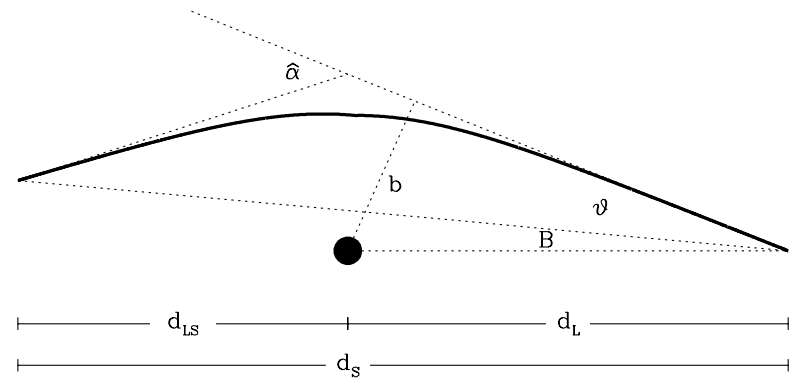

FIG. 1: Schematic diagram of the lensing geometry. Standard quantities are defined as follows: $\mathcal{B}$ is the angular position of the unlensed source; $\vartheta$ is the angular position of an image; $\hat{\alpha}$ is the bending angle; and $d_{L}, d_{S}$, and $d_{L S}$ are angular diameter distances between the observer, lens, and source. The impact parameter $b$ is an invariant of the light ray and is related to the angular image position by $\vartheta=\sin ^{-1}\left(b / d_{L}\right)$.

\section{METRIC AND LIGHT BENDING ANGLE}

We begin by stating our basic assumptions (cf. [4, [5]). Consider a gravitational lens with mass $M_{\bullet}$ that is compact, static, and spherically symmetric, with an asymptotically flat spacetime geometry sufficiently far from the lens [52]. The spacetime is vacuum outside the lens and flat in the absence of the lens. We adopt the standard lensing scenario shown in Figure 1] with the observer and source lying in the asymptotically flat regime of the spacetime.

The light ray's distance of closest approach $r_{0}$ and impact parameter $b$ are both assumed to lie well outside the lens's gravitational radius $\mathrm{m}_{\bullet}=G M_{\bullet} / c^{2}$. The light bending angle is assumed to have the following form at lowest order in $\mathrm{m}_{\bullet}$ :

$$
\hat{\alpha}(b)=A_{1}\left(\frac{\mathrm{m}_{\bullet}}{b}\right)\left(1+\frac{B_{2}}{b^{2}}\right)+\mathcal{O}\left(\frac{\mathrm{m}_{\bullet}}{b}\right)^{2},
$$

where $A_{1}$ and $B_{2}$ are independent of $\mathrm{m}_{\bullet}$ and $b$. Since $b$ and $\mathrm{m}_{\mathbf{0}}$ are invariants of the light ray, this expression for the bending angle is independent of coordinates. If $B_{2} \neq 0$, the bending angle (II) cannot be written as a series in the single parameter $\mathrm{m}_{\bullet} / b$, which places this model outside the PPN framework studied in Papers I and II. We now show that braneworld black holes do yield bending angles of this form.

\section{A. Isotropic coordinates}

The Garriga-Tanaka metric is often written as follows in isotropic coordinates:

$$
\begin{aligned}
d s^{2}= & -\left(1-\frac{2 \mathrm{~m}_{\bullet}}{\bar{r}}-\frac{4 \mathrm{~m}_{\bullet} \ell^{2}}{3 \bar{r}^{3}}\right) d t^{2} \\
& +\left(1+\frac{2 \mathrm{~m}_{\bullet}}{\bar{r}}+\frac{2 \mathrm{~m}_{\bullet} \ell^{2}}{3 \bar{r}^{3}}\right)\left(d \bar{r}^{2}+\bar{r}^{2} d \Omega^{2}\right) .
\end{aligned}
$$


This form is valid only in the limit

$$
\frac{\mathrm{m}_{\bullet}}{\bar{r}} \ll 1, \quad \frac{\ell^{2}}{\bar{r}^{2}} \ll 1,
$$

and the exact metric describing the spacetime geometry around braneworld black holes is not yet known. We shall verify a posteriori that our lensing solutions satisfy (3).

The metric (2) has terms of the form $\pm 2 \mathrm{~m}_{\bullet} / \bar{r}$ as in the weak-deflection limit of general relativity. Braneworld effects enter via the $\ell$ terms. (When $\ell=0$ we recover the standard far-field black hole metric of general relativity.) Notice that the $\ell$ terms factor as $\left(\mathrm{m}_{\bullet} / \bar{r}\right)\left(\ell^{2} / \bar{r}^{2}\right)$, so we can adopt the approach of taking Taylor series expansions in $\mathrm{m}_{\bullet} / \bar{r}$ to obtain the appropriate weak-deflection limit including the braneworld terms. Later we shall consider when it is appropriate to take series expansions in $\ell / \bar{r}$ as well.

We can think of the metric (2) more generally as having the form

$$
d s^{2}=-\overline{\mathcal{A}}(\bar{r}) d t^{2}+\overline{\mathcal{B}}(\bar{r}) d \bar{r}^{2}+\overline{\mathcal{C}}(\bar{r}) \bar{r}^{2} d \Omega^{2},
$$

where the metric functions $\overline{\mathcal{A}}(\bar{r})$ and $\overline{\mathcal{B}}(\bar{r})=\overline{\mathcal{C}}(\bar{r})$ are readily identified. For such a metric, the distance of closest approach $\bar{r}_{0}$ is related to the impact parameter $b$ by (cf. eq. 12 of Paper I)

$$
\begin{aligned}
b & =\bar{r}_{0}\left[\frac{\overline{\mathcal{C}}\left(\bar{r}_{0}\right)}{\overline{\mathcal{A}}\left(\bar{r}_{0}\right)}\right]^{1 / 2} \\
& =\bar{r}_{0}\left[1+\left(2+\frac{\ell^{2}}{\bar{r}_{0}^{2}}\right) \frac{\mathrm{m}_{\bullet}}{\bar{r}_{0}}+\mathcal{O}\left(\frac{\mathrm{m}_{\bullet}}{\bar{r}_{0}}\right)^{2}\right] .
\end{aligned}
$$

Inverting this relation yields

$$
\bar{r}_{0}=b\left[1-\left(2+\frac{\ell^{2}}{b^{2}}\right) \frac{\mathrm{m}_{\bullet}}{b}+\mathcal{O}\left(\frac{\mathrm{m}_{\bullet}}{b}\right)^{2}\right] .
$$

For a metric of the form (4), the light bending angle can be written as

$$
\hat{\alpha}\left(\bar{r}_{0}\right)=2 \int_{\bar{r}_{0}}^{\infty} \frac{1}{\bar{r}^{2}}\left[\frac{\overline{\mathcal{A}} \overline{\mathcal{B}}}{\overline{\mathcal{C}}^{2} / b^{2}-\overline{\mathcal{A}} \overline{\mathcal{C}} / \bar{r}^{2}}\right]^{1 / 2} d \bar{r}-\pi .
$$

Plugging in the metric functions, and temporarily replacing $b$ with $\bar{r}_{0}$ using (5), we can write a series expansion for the integrand,

$$
\hat{\alpha}\left(\bar{r}_{0}\right)=2 \int_{\bar{r}_{0}}^{\infty} \frac{\bar{r}_{0}}{\bar{r}\left(\bar{r}^{2}-\bar{r}_{0}^{2}\right)^{1 / 2}}\left[1+\frac{2 \bar{r}^{2} \bar{r}_{0}^{2}+\ell^{2}\left(\bar{r}^{2}+\bar{r}_{0}+\bar{r}_{0}^{2}\right)}{\bar{r}_{0}^{2}\left(\bar{r}+\bar{r}_{0}\right)} \frac{\mathrm{m}_{\bullet}}{\bar{r}_{0}}+\mathcal{O}\left(\frac{\mathrm{m}_{\bullet}}{\bar{r}_{0}}\right)^{2}\right] d \bar{r}-\pi
$$

Carrying out the integration yields the deflection angle in terms of the isotropic coordinate distance of closest approach,

$$
\hat{\alpha}\left(\bar{r}_{0}\right)=4 \frac{\mathrm{m}_{\bullet}}{\bar{r}_{0}}\left(1+\frac{\ell^{2}}{\bar{r}_{0}^{2}}\right)+\mathcal{O}\left(\frac{\mathrm{m}_{\bullet}}{\bar{r}_{0}}\right)^{2} .
$$

This agrees with the bending angle found by Kar \& Sinha 21. However, as emphasized in Paper I, expressions like (9) are coordinate dependent and should be re-expressed in invariant form. Using (6) to rewrite the distance of closest approach $\bar{r}_{0}$ in terms of the invariant impact parameter $b$ yields

$$
\hat{\alpha}(b)=4 \frac{\mathrm{m}_{\bullet}}{b}\left(1+\frac{\ell^{2}}{b^{2}}\right)+\mathcal{O}\left(\frac{\mathrm{m}_{\bullet}}{b}\right)^{2} .
$$

At this order of approximation the form of the bending angle is the same for $r_{0}$ and $b$; still, it is important to use the invariant expression. Notice that (10) has the form assumed in (1), with $A_{1}=4$ and $B_{2}=\ell^{2}$, which shows that braneworld black hole lensing lies outside the standard PPN framework.

\section{B. Standard coordinates}

As a consistency check of (10), and to connect with our formalism in Papers I and II, we rederive the bending angle starting from standard coordinates (or the area gauge [20]). In this case we write the metric in the form

$$
d s^{2}=-A(r) d t^{2}+B(r) d r^{2}+r^{2} d \Omega^{2} .
$$

Comparing the $d \Omega^{2}$ terms in (2) and (11) shows that the isotropic and standard radial coordinates are related by

$$
r=\bar{r}\left[1+\left(1+\frac{\ell^{2}}{3 \bar{r}^{2}}\right) \frac{\mathrm{m}_{\bullet}}{\bar{r}}+\mathcal{O}\left(\frac{\mathrm{m}_{\bullet}}{\bar{r}}\right)^{2}\right] .
$$

Inverting this relation yields

$$
\bar{r}=r\left[1-\left(1+\frac{\ell^{2}}{3 r^{2}}\right) \frac{\mathrm{m}_{\bullet}}{r}+\mathcal{O}\left(\frac{\mathrm{m}_{\bullet}}{r}\right)^{2}\right] .
$$

Returning to the metric (2) and changing radial coordinates yields a metric of the form (11) with metric functions

$$
\begin{aligned}
& A(r)=1-2\left(1+\frac{2 \ell^{2}}{3 r^{2}}\right) \frac{\mathrm{m}_{\bullet}}{r}+\mathcal{O}\left(\frac{\mathrm{m}_{\bullet}}{r}\right)^{2}, \\
& B(r)=1+2\left(1+\frac{\ell^{2}}{r^{2}}\right) \frac{\mathrm{m}_{\bullet}}{r}+\mathcal{O}\left(\frac{\mathrm{m}_{\bullet}}{r}\right)^{2} .
\end{aligned}
$$


When the metric is written in the form (11), the standard-coordinate distance of closest approach $r_{0}$ and invariant impact parameter $b$ are related by

$$
\begin{aligned}
b & =\frac{r_{0}}{\sqrt{A\left(r_{0}\right)}} \\
& =r_{0}\left[1+\left(1+\frac{2 \ell^{2}}{3 r_{0}^{2}}\right) \frac{\mathrm{m}_{\bullet}}{r_{0}}+\mathcal{O}\left(\frac{\mathrm{m}_{\bullet}}{r_{0}}\right)^{2}\right], \\
r_{0} & =b\left[1-\left(1+\frac{2 \ell^{2}}{3 b^{2}}\right) \frac{\mathrm{m}_{\bullet}}{b}+\mathcal{O}\left(\frac{\mathrm{m}_{\bullet}}{b}\right)^{2}\right] .
\end{aligned}
$$

The light bending angle can be written as

$$
\hat{\alpha}\left(r_{0}\right)=2 \int_{r_{0}}^{\infty} \frac{1}{r^{2}}\left[\frac{\bar{A} \bar{B}}{1 / b^{2}-\bar{A} / r^{2}}\right]^{1 / 2} d r-\pi .
$$

Plugging in the metric functions, and temporarily replacing $b$ with $r_{0}$ using (17), we can write a series expansion for the integrand,

$$
\begin{aligned}
\hat{\alpha}\left(r_{0}\right)=2 \int_{r_{0}}^{\infty} \frac{r_{0}}{r\left(r^{2}-r_{0}^{2}\right)^{1 / 2}} & {\left[1+\frac{3 r^{2} r_{0}^{2}\left(r^{2}+r r_{0}+r_{0}^{2}\right)+\ell^{2}\left(2 r^{4}+2 r^{3} r_{0}+2 r^{2} r_{0}^{2}+3 r r_{0}^{3}+3 r_{0}^{4}\right)}{3 r^{3} r_{0}^{2}\left(r+r_{0}\right)} \frac{\mathrm{m}_{\bullet}}{r_{0}}\right.} \\
& \left.+\mathcal{O}\left(\frac{\mathrm{m}_{\bullet}}{r_{0}}\right)^{2}\right] d r-\pi .
\end{aligned}
$$

Carrying out the integration yields the deflection angle in terms of the coordinate distance of closest approach,

$$
\hat{\alpha}\left(r_{0}\right)=4 \frac{\mathrm{m}_{\bullet}}{r_{0}}\left(1+\frac{\ell^{2}}{r_{0}^{2}}\right)+\mathcal{O}\left(\frac{\mathrm{m}_{\bullet}}{r_{0}}\right)^{2} .
$$

Using (17) to rewrite the distance of closest approach $r_{0}$ in terms of the impact parameter $b$ yields

$$
\hat{\alpha}(b)=4 \frac{\mathrm{m}_{\bullet}}{b}\left(1+\frac{\ell^{2}}{b^{2}}\right)+\mathcal{O}\left(\frac{\mathrm{m}_{\bullet}}{b}\right)^{2} .
$$

This result agrees with (10), showing that we arrive at the same desired invariant bending angle expression starting from isotropic and standard coordinates.

\section{IMAGE POSITIONS, MAGNIFICATIONS, AND CENTROID}

We now go beyond the bending angle to determine observable quantities in braneworld black hole lensing. We examine the image positions and magnifications in this section, and defer the time delay to Section $[\mathbf{T}$ (This analysis parallels Section IV in Paper I.) We begin with the general lens equation (cf. Figure 1),

$$
\tan \mathcal{B}=\tan \vartheta-D(\tan \vartheta+\tan (\hat{\alpha}-\vartheta))
$$

where $\mathcal{B}$ is the angular position of the source, $\vartheta=$ $\sin ^{-1}\left(b / d_{L}\right)$ is the angular position of the image, and $D=d_{L S} / d_{S}$. We shall see that the lens equation yields two images in the far-field (weak-deflection) regime, one on the same side of the lens as the source and the other on the opposite side. Following the convention of Papers I and II, angles describing image positions are taken to be positive. This forces the source's angular position to have different signs: $\mathcal{B}$ is positive when the image is on the same side of the lens as the source (as depicted in Figure 1); while $\mathcal{B}$ is negative when the image is on the opposite of the lens from the source.

We now seek an appropriate series expansion of the lens equation in the weak-deflection limit. First, we change variables in light of the fact that lensing quantities naturally scale with the weak-deflection angular Einstein ring radius,

$$
\vartheta_{E}=\sqrt{\frac{4 G M_{\bullet} d_{L S}}{c^{2} d_{L} d_{S}}}
$$

Specifically, we define:

$$
\beta=\frac{\mathcal{B}}{\vartheta_{E}}, \quad \theta=\frac{\vartheta}{\vartheta_{E}}, \quad \varepsilon_{m}=\frac{\vartheta_{\bullet}}{\vartheta_{E}}=\frac{\vartheta_{E}}{4 D},
$$

where $\vartheta_{\bullet}=\tan ^{-1}\left(\mathrm{~m}_{\bullet} / d_{L}\right)$. In other words, the quantities $\beta$ and $\theta$ are the scaled angular positions of the source and image, respectively. The quantity $\varepsilon_{m}$ represents the angle subtended by the gravitational radius normalized by the angular Einstein radius, and it replaces $\mathrm{m}_{\bullet} / b$ as our expansion parameter. We also define an angle associated with the braneworld length scale $\ell$,

$$
\vartheta_{\ell}=\tan ^{-1}\left(\frac{\ell}{d_{L}}\right)
$$

and a scaled version of this angle

$$
\varepsilon_{\ell}=\frac{\vartheta_{\ell}}{\vartheta_{E}} .
$$

When it becomes appropriate to consider series expansions in $\ell / b$ (below), we shall actually use $\varepsilon_{\ell}$ as the expansion parameter. The conditions (3) for validity of the 
Garriga-Tanaka metric are equivalent to the conditions $\varepsilon_{m} \ll 1$ and $\varepsilon_{\ell} \ll 1$.

With these substitutions, and the bending angle from (21), the lens equation becomes

$$
0=\left[-\beta+\theta-\frac{1}{\theta}\left(1+\frac{\varepsilon_{\ell}^{2}}{\theta^{2}}\right)\right] \varepsilon_{m}+\mathcal{O}\left(\varepsilon_{m}\right)^{2} .
$$

To determine braneworld effects on the image positions, we then postulate that the position can be expanded in the form

$$
\theta=\theta_{0}+\theta_{1} \varepsilon_{\ell}+\theta_{2} \varepsilon_{\ell}^{2}+\mathcal{O}\left(\varepsilon_{\ell}\right)^{3} .
$$

Plugging this into (27) yields

$$
\begin{aligned}
0= & \left(-\beta+\theta_{0}-\frac{1}{\theta_{0}}\right)+\left(1+\frac{1}{\theta_{0}^{2}}\right) \theta_{1} \varepsilon_{\ell} \\
& +\frac{\left(1+\theta_{0}^{2}\right) \theta_{0} \theta_{2}-1-\theta_{1}^{2}}{\theta_{0}^{3}} \varepsilon_{\ell}^{2}+\mathcal{O}\left(\varepsilon_{\ell}\right)^{3}
\end{aligned}
$$

This is the desired series expansion of the lens equation.

The zeroth-order term in (29) is just the usual lens equation for the weak-deflection limit of general relativity, whose solution is

$$
\theta_{0}=\frac{1}{2}\left(\sqrt{\beta^{2}+4}+\beta\right)
$$

We neglect the negative solution because by convention angles describing image positions are positive. The positive-parity image $\theta_{0}^{+}$, which lies on the same side of the lens as the source, is found by using $\beta>0$. The negative-parity image $\theta_{0}^{-}$is then found by using $\beta<0$. Explicitly,

$$
\theta_{0}^{ \pm}=\frac{1}{2}\left(\sqrt{4+\beta^{2}} \pm|\beta|\right) .
$$

The first-order term in (29) can be satisfied only if $\theta_{1}=0$. In other words, there is no braneworld correction to the lensed image positions at first order in $\varepsilon_{\ell} \sim \ell / b$. This is not surprising, since braneworld effects enter the metric at order $(\ell / r)^{2}$.

The second-order term in (29) is satisfied if

$$
\theta_{2}=\frac{1}{\theta_{0}\left(1+\theta_{0}^{2}\right)} \text {. }
$$

Thus, the full expression for the image position in braneworld gravity is

$$
\theta=\theta_{0}+\frac{\varepsilon_{\ell}^{2}}{\theta_{0}\left(1+\theta_{0}^{2}\right)}+\mathcal{O}\left(\varepsilon_{\ell}\right)^{3}
$$

Rewriting $\theta_{0}$ in terms of $\beta$ using (31), we can express the image positions in terms of the source position as

$$
\begin{aligned}
\theta^{ \pm}= & \frac{1}{2}\left(\sqrt{4+\beta^{2}} \pm|\beta|\right) \\
& +\frac{1}{2}\left(\frac{2+\beta^{2}}{\sqrt{4+\beta^{2}}} \mp|\beta|\right) \varepsilon_{\ell}^{2}+\mathcal{O}\left(\varepsilon_{\ell}\right)^{3} .
\end{aligned}
$$

The coefficient of $\varepsilon_{\ell}^{2}$ is positive for all value of $\beta$, which means that braneworld effects push both the positiveand negative-parity images farther away from the lens (relative to the results for general relativity).

By spherical symmetry, the signed magnification $\mu$ of a lensed image at angular position $\vartheta$ is

$$
\mu(\vartheta)=\left[\frac{\sin \mathcal{B}(\vartheta)}{\sin \vartheta} \frac{d \mathcal{B}(\vartheta)}{d \vartheta}\right]^{-1}
$$

After changing to our scaled variables, we first make a Taylor series expansion in $\varepsilon_{m}$ :

$$
\mu=\frac{\theta^{8}}{\left(\theta^{4}+\theta^{2}+3 \varepsilon_{\ell}^{2}\right)\left(\theta^{4}-\theta^{2}-\varepsilon_{\ell}^{2}\right)}+\mathcal{O}\left(\varepsilon_{m}\right) .
$$

Now using (33) for the image position, we find

$$
\mu=\frac{\theta_{0}^{4}}{\theta_{0}^{4}-1}-\frac{2 \theta_{0}^{4}}{\left(\theta_{0}^{4}-1\right)\left(\theta_{0}^{2}+1\right)^{3}} \varepsilon_{\ell}^{2}+\mathcal{O}\left(\varepsilon_{\ell}\right)^{3} .
$$

The absolute magnifications in terms of the source position are given by

$$
\begin{aligned}
\left|\mu^{ \pm}\right|= & \frac{1}{2}\left(\frac{2+\beta^{2}}{|\beta| \sqrt{4+\beta^{2}}} \pm 1\right) \\
& -\frac{2}{|\beta|\left(4+\beta^{2}\right)^{3 / 2}} \varepsilon_{\ell}^{2}+\mathcal{O}\left(\varepsilon_{\ell}\right)^{3}
\end{aligned}
$$

where $\mu^{-}<0$. Observe that at lowest order the braneworld magnifications have $\mu^{+}+\mu^{-}=1$. This is identical to the lowest-order universal magnification relation in eq. (36) of Paper II. It is interesting to see that braneworld gravity obeys the relation originally derived for PPN models, even though it lies outside the PPN framework.

In cases where the two images cannot be separately resolved (such as microlensing [3, 31, 32]), the useful quantities are the total magnification and the magnificationweighted centroid position. The total magnification can be written as

$$
\begin{aligned}
\mu_{\mathrm{tot}} & \equiv\left|\mu^{+}\right|+\left|\mu^{-}\right| \\
& =\frac{2+\beta^{2}}{|\beta| \sqrt{4+\beta^{2}}}-\frac{4}{|\beta|\left(4+\beta^{2}\right)^{3 / 2}} \varepsilon_{\ell}^{2}+\mathcal{O}\left(\varepsilon_{\ell}\right)^{3}
\end{aligned}
$$

The magnification-weighted centroid position can be written as

$$
\begin{aligned}
\Theta_{\mathrm{cent}} & \equiv \frac{\theta^{+}\left|\mu^{+}\right|-\theta^{-}\left|\mu^{-}\right|}{\left|\mu^{+}\right|+\left|\mu^{-}\right|} \\
& =\frac{|\beta|\left(3+\beta^{2}\right)}{2+\beta^{2}}+\frac{2|\beta|}{\left(2+\beta^{2}\right)^{2}} \varepsilon_{\ell}^{2}+\mathcal{O}\left(\varepsilon_{\ell}\right)^{3}
\end{aligned}
$$

We see that braneworld effects decrease the total magnification and push the centroid farther from the lens (compared with the results for general relativity). 


\section{TIME DELAY}

We now derive the lensing time delay (in parallel with Section V of Paper I). We first focus on a spacetime that is static and asymptotically flat, and discuss the generalization to a curved universe cosmology at the end of this section.

Let $R_{\mathrm{src}}$ and $R_{\mathrm{obs}}$ be the radial coordinates of the source and observer, respectively. From geometry relative to the flat metric of the distant observer (who is assumed to be at rest in the natural coordinates of the metric eq. 11), we can work out (see Figure 1)

$$
R_{\mathrm{obs}}=d_{L}, \quad R_{\mathrm{src}}=\left(d_{L S}^{2}+d_{S}^{2} \tan ^{2} \mathcal{B}\right)^{1 / 2} .
$$

The radial distances are very nearly the same as angular diameter distances since the source and observer are in the asymptotically flat region of the spacetime. In other words, the distortions in distances near the black hole are assumed to have little impact on the total flat met- ric distance from the compact body to the observer or source.

In the absence of the lens the light ray would travel along a linear path from the source to the observer with length $d_{S} / \cos \mathcal{B}$. The time delay $\tau$ is the difference between the light travel time for the actual ray, and the travel time for the straight line the ray would have taken in the absence of the lens. This can be written as

$$
c \tau=\mathcal{T}\left(R_{\mathrm{src}}\right)+\mathcal{T}\left(R_{\mathrm{obs}}\right)-\frac{d_{S}}{\cos \mathcal{B}}
$$

with (see eq. 91 of Paper I)

$$
\mathcal{T}(R)=\frac{1}{b} \int_{r_{0}}^{R} \frac{1}{A(r)} \sqrt{\frac{A(r) B(r)}{1 / b^{2}-A(r) / r^{2}}} d r
$$

Using the metric functions (14) and (15), we find for braneworld gravity

$$
\begin{aligned}
\mathcal{T}(R) & =\int_{r_{0}}^{R} \frac{r}{\sqrt{r^{2}-r_{0}^{2}}}\left[1+\frac{3 r^{2} r_{0}\left(2 r+3 r_{0}\right)+\ell^{2}\left(2 r^{2}+7 r r_{0}+7 r_{0}^{2}\right)}{3 r^{3} r_{0}\left(r+r_{0}\right)} \frac{\mathrm{m}_{\bullet}}{r_{0}}+\mathcal{O}\left(\frac{\mathrm{m}_{\bullet}}{r_{0}}\right)^{2}\right] d r \\
& =\sqrt{R^{2}-r_{0}^{2}}+\left[2 \ln \left(\frac{R+\sqrt{R^{2}-r_{0}^{2}}}{r_{0}}\right)+\sqrt{R-r_{0}}\left(1+\ell^{2} \frac{9 R+7 r_{0}}{3 R r_{0}^{2}}\right)\right]+\mathcal{O}\left(\frac{\mathrm{m}_{\bullet}}{r_{0}}\right)^{2}
\end{aligned}
$$

To obtain a coordinate-invariant expression, we could replace $r_{0}$ with $b$ using (17). We would then want to take a series expansion in $b / R$ as well as $\mathrm{m}_{\bullet} / b$, because they are of the same order (see Section V of Paper I).

It is simpler to proceed directly to the time delay expressed in terms of our scaled angular variables. We compute $\mathcal{T}\left(R_{\text {src }}\right)$ and $\mathcal{T}\left(R_{\text {obs }}\right)$ using the radii from (41). We change to angular variables using $b=d_{L} \sin \vartheta$, and then reintroduce the scaled angular variables $\theta$ and $\beta$ defined in (24). We work to lowest order in $\varepsilon_{m}$, and then take a Taylor series in the dimensionless braneworld parameter $\varepsilon_{\ell}$. The result is

$$
\frac{\tau}{\tau_{E}}=\frac{1}{2}\left[1+\beta^{2}-\theta_{0}^{2}-\ln \left(\frac{d_{L} \theta_{0}^{2} \vartheta_{E}^{2}}{4 d_{L S}}\right)\right]+\frac{\varepsilon_{\ell}^{2}}{2 \theta_{0}^{2}}+\mathcal{O}\left(\varepsilon_{\ell}\right)^{3},
$$

where the natural time scale is

$$
\tau_{E} \equiv \frac{d_{L} d_{S}}{c d_{L S}} \vartheta_{E}^{2}=4 \frac{\mathrm{m}_{\bullet}}{c}
$$

Note that we have used (33) for the image position to obtain an expression written in terms of $\theta_{0}$, the image position in the weak-deflection limit of general relativity.

More interesting than the individual time delays is the differential delay $\Delta \tau=\tau^{-}-\tau^{+}$between the negative- and positive-parity images:

$$
\begin{aligned}
\frac{\Delta \tau}{\tau_{E}}= & {\left[\frac{\left(\theta_{0}^{+}\right)^{2}-\left(\theta_{0}^{-}\right)^{2}}{2}+\ln \frac{\theta_{0}^{+}}{\theta_{0}^{-}}\right] } \\
& +\frac{\varepsilon_{\ell}^{2}}{2}\left[\left(\theta_{0}^{-}\right)^{-2}-\left(\theta_{0}^{+}\right)^{-2}\right]+\mathcal{O}\left(\varepsilon_{\ell}\right)^{3} \\
= & {\left[\frac{1}{2}|\beta| \sqrt{4+\beta^{2}}+\ln \left(\frac{\sqrt{4+\beta^{2}}+|\beta|}{\sqrt{4+\beta^{2}}-|\beta|}\right)\right] } \\
& +\frac{\varepsilon_{\ell}^{2}}{2}|\beta| \sqrt{4+\beta^{2}}+\mathcal{O}\left(\varepsilon_{\ell}\right)^{3} .
\end{aligned}
$$

The first expression is written in terms of the image positions $\theta_{0}^{ \pm}$, while the second is written in terms of the source position. In each case, the order unity term recovers the familiar time delay in the weak-deflection limit of general relativity. To simplify the notation below, we write the differential time delay as

$$
\frac{\Delta \tau}{\tau_{E}}=\Lambda_{0}+\Lambda_{1}+\Lambda_{0} \varepsilon_{\ell}^{2}+\mathcal{O}\left(\varepsilon_{\ell}\right)^{3}
$$

where

$$
\Lambda_{0}=\frac{1}{2}|\beta| \sqrt{4+\beta^{2}}, \quad \Lambda_{1}=\ln \left(\frac{\sqrt{4+\beta^{2}}+|\beta|}{\sqrt{4+\beta^{2}}-|\beta|}\right) .
$$


It is straightforward to place this analysis in a background universe that is curved and expanding. We are working in the limit that the impact parameter is small compared to the distances between the observer, lens, and source. Thus, the light path is determined by the background cosmology for all but the tiny fraction of the path when it is near the lens. (See [1, 2] for further discussion.) All of the previous analysis holds if we interpret $d_{L}, d_{S}$, and $d_{L S}$ as cosmological angular diameter distances, and we modify the natural time scale from (46) to

$$
\tau_{E} \equiv\left(1+z_{L}\right) \frac{d_{L} d_{S}}{c d_{L S}} \vartheta_{E}^{2}=4\left(1+z_{L}\right) \frac{\mathrm{m}_{\bullet}}{c},
$$

where $z_{L}$ is the cosmological redshift of the lens.

\section{SEMI-CLASSICAL INTERFERENCE OPTICS}

The foregoing analysis applies in the limit of geometric optics. We now examine how the wave nature of light can affect gravitational lensing observables. Wave optics have been studied for standard weak-deflection lensing in general relativity (see the review by Nakamura \& Deguchi 27], and Sections 4.7 and Chapter 7 of Schneider et al. 1]), but to our knowledge have not been treated before in the braneworld lensing literature.

In analogy with the Young double-slit experiment, we may consider that light waves from the positiveand negative-parity images interact to produce an interference pattern at the observer. To quantify different regimes, suppose a pointlike light source emits a monochromatic, spherical light wave of period $T=2 \pi / \omega$ or wavelength $\lambda=c T$, which is lensed by a braneworld black hole. Geometric optics apply in the limit $T \rightarrow 0$. If the period is finite but small compared with the time delay between the images $(T \ll \Delta \tau)$, then we are in the semi-classical limit (cf. 27, 38]). This is the regime we shall investigate. Larger periods $T \gtrsim \Delta \tau$ lead to the physical optics limit. In this regime, all regions of the lens plane contribute to interference effects. The analysis therefore requires knowledge of the time delay function across the entire lens plane, which in turn requires knowledge of the metric at all such positions. However, the full metric for braneworld black holes is not yet known, which means that we cannot give a complete wave optics treatment of lensing in braneworld gravity at this time.

There is a well-established connection between the geometric optics and semi-classical limits. If geometric optics predict a set of images with magnifications $\mu_{i}$ and time delays $\Delta \tau_{i j}$, then the total magnification in the semi-classical limit is given as follows (e.g., Sections 4.7 and 7.1 of [1], and Section 2 of [27]):

$$
\mathcal{M}=\sum_{j}\left|\mu_{j}\right|+2 \sum_{i<j}\left|\mu_{i} \mu_{j}\right|^{1 / 2} \cos \left[\omega \Delta \tau_{j i}-\left(n_{j}-n_{i}\right) \frac{\pi}{2}\right] .
$$

where $n_{k}=0,1,2$ depending on whether the $k$-th image is a minimum, saddle, or maximum, respectively. The first sum gives the total magnification in the geometric optics limit, while the second captures the corrections due to semi-classical interference between the images. There is a phase factor of $\omega \Delta \tau_{i j}$ from the lensing time delay, and an additional factor of $\left(n_{j}-n_{i}\right) \pi / 2$ from differences in the phases of the images upon exiting the lens plane.

Lensing by a black hole produces a positive and negative image pair, so we have $n_{12}=1-0=1$ and $\Delta \tau_{12}=\tau^{-}-\tau^{+}$. The total magnification in the semiclassical limit can then be written as

$$
\mathcal{M}=\left|\mu^{+}\right|+\left|\mu^{-}\right|+2\left|\mu^{+} \mu^{-}\right|^{1 / 2} \sin (\omega \Delta \tau) .
$$

The sine term creates a series of bright and dark fringes in the magnification as a function of energy or wavelength. Bright fringes (maxima in $\mathcal{M}$ ) occur when the phase difference is $\omega \Delta \tau=2 j \pi+\pi / 2$ for integer $j$, which correspond to photon energies

$$
E_{j}^{\mathrm{br}}=(j+1 / 4) \frac{h}{\Delta \tau}, \quad j=0,1,2, \ldots
$$

Dark fringes (minima in $\mathcal{M}$ ) occur when the phase difference is $\omega \Delta \tau=(2 j+1) \pi+\pi / 2$, or energies

$$
E_{j}^{\mathrm{dk}}=(j+3 / 4) \frac{h}{\Delta \tau}, \quad j=0,1,2, \ldots
$$

Note that the energy spacing between adjacent bright fringes, or adjacent dark fringes, is always $\Delta E=h / \Delta \tau$, independent of $j$.

For a braneworld black hole, the magnification sum $\left|\mu^{+}\right|+\left|\mu^{-}\right|$is given in (39), and the magnification product is

$$
\left|\mu^{+} \mu^{-}\right|^{1 / 2}=\frac{1}{|\beta| \sqrt{4+\beta^{2}}}-\frac{2+\beta^{2}}{|\beta|\left(4+\beta^{2}\right)^{3 / 2}} \varepsilon_{\ell}^{2}+\mathcal{O}\left(\varepsilon_{\ell}\right)^{3} .
$$

Consequently, the semi-classical total magnification is given as follows in terms of the source position:

$$
\begin{aligned}
\mathcal{M}= & \frac{\sqrt{4+\beta^{2}}}{|\beta|}\left[\frac{2+\beta^{2}+2 \sin (\omega \Delta \tau)}{4+\beta^{2}}\right. \\
& \left.-\frac{4+2\left(2+\beta^{2}\right) \sin (\omega \Delta \tau)}{\left(4+\beta^{2}\right)^{2}} \varepsilon_{\ell}^{2}+\mathcal{O}\left(\varepsilon_{\ell}\right)^{3}\right] .
\end{aligned}
$$

Using the time delay from (49), we find that the bright fringes have magnification

$$
\mathcal{M}^{\mathrm{br}}=\frac{\sqrt{4+\beta^{2}}}{|\beta|}\left[1-\frac{2}{4+\beta^{2}} \varepsilon_{\ell}^{2}+\mathcal{O}\left(\varepsilon_{\ell}\right)^{3}\right],
$$

and are located at energies

$$
E_{j}^{\mathrm{br}}=\frac{(j+1 / 4) h}{\left(\Lambda_{0}+\Lambda_{1}\right) \tau_{E}}\left[1-\frac{\Lambda_{0}}{\Lambda_{0}+\Lambda_{1}} \varepsilon_{\ell}^{2}+\mathcal{O}\left(\varepsilon_{\ell}\right)^{3}\right],
$$


while the dark fringes have magnification

$$
\mathcal{M}^{\mathrm{dk}}=\frac{|\beta|}{\sqrt{4+\beta^{2}}}\left[1+\frac{2}{4+\beta^{2}} \varepsilon_{\ell}^{2}+\mathcal{O}\left(\varepsilon_{\ell}\right)^{3}\right],
$$

and are located at energies

$$
E_{j}^{\mathrm{dk}}=\frac{(j+3 / 4) h}{\left(\Lambda_{0}+\Lambda_{1}\right) \tau_{E}}\left[1-\frac{\Lambda_{0}}{\Lambda_{0}+\Lambda_{1}} \varepsilon_{\ell}^{2}+\mathcal{O}\left(\varepsilon_{\ell}\right)^{3}\right],
$$

The absolute peak-to-trough distance between bright and dark fringes is

$\mathcal{M}^{\mathrm{br}}-\mathcal{M}^{\mathrm{dk}}=\frac{4}{|\beta| \sqrt{4+\beta^{2}}}\left[1-\frac{2+\beta^{2}}{4+\beta^{2}} \varepsilon_{\ell}^{2}+\mathcal{O}\left(\varepsilon_{\ell}\right)^{3}\right]$,

while the fractional difference is

$$
\frac{\mathcal{M}^{\mathrm{br}}-\mathcal{M}^{\mathrm{dk}}}{\mathcal{M}^{\mathrm{br}}+\mathcal{M}^{\mathrm{dk}}}=\frac{2}{2+\beta^{2}}\left[1-\frac{\beta^{2}}{2+\beta^{2}} \varepsilon_{\ell}^{2}+\mathcal{O}\left(\varepsilon_{\ell}\right)^{3}\right] .
$$

We see that braneworld effects (from the extra dimension of space) shift the interference fringes to lower energies, and contract the energy spacing $E_{j}^{\mathrm{dk}}-E_{j}^{\mathrm{br}}$. Braneworld effects also reduce the magnification of the bright fringes and increase the magnification of the dark fringes, which reduces the peak-to-trough distance in the fringe pattern.

\section{LARGE BRANEWORLD BLACK HOLES IN TRADITIONAL LENSING SCENARIOS}

We have seen that all the braneworld corrections to weak-deflection black hole lensing scale with

$\varepsilon_{\ell}^{2}=\left[\frac{\tan ^{-1}\left(\ell / d_{L}\right)}{\vartheta_{E}}\right]^{2} \approx \frac{\ell^{2} c^{2}}{4 G M_{\bullet}} \frac{d_{S}}{d_{L} d_{L S}}=\frac{\ell^{2}}{4 \mathrm{~m}_{\bullet}} \frac{d_{S}}{d_{L} d_{L S}}$,

where we used $\tan ^{-1}\left(\ell / d_{L}\right) \approx \ell / d_{L}$ and the definition of $\vartheta_{E}$ from (23). Notice that $\varepsilon_{\ell}$ is given by the ratio of the braneworld scale $\ell$ to the geometric mean of the gravitational scale $\mathrm{m}_{\bullet}$ and the astrophysical distance scale.

There are two ways to maximize braneworld effects. One is to consider a small black hole mass, since $\varepsilon_{\ell}^{2} \propto$ $\mathrm{m}_{\bullet}^{-1}$. The other possibility is to make either $d_{L}$ or $d_{L S}$ small. Notice that if $d_{L} \ll d_{S}$ then $d_{L S} \approx d_{S}$ and the combination of distances reduces to $1 / d_{L}$. Alternatively, if $d_{L S} \ll d_{S}$ then $d_{L} \approx d_{S}$ and the combination of distances reduces to $1 / d_{L S}$. We can combine these two possibilities and write

$$
\varepsilon_{\ell}^{2} \approx \frac{\ell^{2}}{4 \mathrm{~m}_{\bullet} d_{\min }}, \quad d_{\min } \equiv \min \left(d_{L}, d_{L S}\right) \ll d_{S} .
$$

Remarkably, $\varepsilon_{\ell}^{2}$ does not depend on the distance to the source if $d_{S} \gg d_{\min }$.

We now examine traditional astrophysical scenarios in which lensing is or may be observed, and assess whether braneworld effects are likely to be detectable. We shall see that all of the scenarios have $\varepsilon_{\ell} \ll 1$, so the condition (3) is satisfied and our use of the Garriga-Tanaka metric is valid.

Primoridal braneworld black holes may grow to supermassive scales by accreting dark energy [28]. Therefore let us consider lensing by the supermassive black hole at the center of our Galaxy. The black hole has a mass of $M_{\bullet}=(3.6 \pm 0.2) \times 10^{6} M_{\odot}$ [29], corresponding to $\mathrm{m}_{\mathbf{0}}=5.3 \times 10^{11} \mathrm{~cm}=1.7 \times 10^{-7} \mathrm{pc}$, and a distance $d_{L}=7.9 \pm 0.4 \mathrm{kpc} 30$. Assuming that the lens-source distance $d_{L S}$ is much smaller than the observer-lens distance $d_{L}$, eq. (65) yields

$$
\varepsilon_{\ell}^{2} \approx 6.1 \times 10^{-35} \times\left(\frac{\ell}{0.2 \mathrm{~mm}}\right)^{2}\left(\frac{d_{L S}}{\mathrm{pc}}\right)^{-1} .
$$

It would be very challenging to measure braneworld effects in lensing by the Galactic black hole.

To increase $\varepsilon_{\ell}^{2}$ we need to lower the black hole mass. Considering stellar-mass black holes brings us into the realm of microlensing (e.g., 3, 31, 32]). In Galactic microlensing the typical distances between observer, lens, and source are kiloparsecs, so eq. (64) yields

$$
\varepsilon_{\ell}^{2} \approx 2.2 \times 10^{-31} \times\left(\frac{\ell}{0.2 \mathrm{~mm}}\right)^{2}\left(\frac{M_{\bullet}}{M_{\odot}}\right)^{-1}\left(\frac{d_{S}}{d_{L} d_{L S}}\right),
$$

where the distances $d_{i}$ are in kpc. Compared with the example of the Galactic supermassive black hole, we have gained six orders of magnitude in mass but lost three in distance, so the braneworld effects are still very small.

An intriguing microlensing system is the binary pulsar J0737-3039 33]. The binary orbit is seen nearly edge-on, so that when one neutron star passes behind the other in projection there may be significant lensing effects [34]. Although the lens in this case is not a black hole, it is still a compact object to which our formalism applies. The Einstein radius in this system is 2550 $\mathrm{km}$ while the gravitational radius is $\mathrm{m}_{\bullet}=1.85 \mathrm{~km}$ [34], so the weak-deflection regime applies. The lens-source distance is given by the semimajor axis of the orbit, $d_{L S} \approx a=8.79 \times 10^{5} \mathrm{~km}$, and is much smaller than the distance to the lens, so eq. (65) yields

$$
\varepsilon_{\ell}^{2} \approx 6.1 \times 10^{-21} \times\left(\frac{\ell}{0.2 \mathrm{~mm}}\right)^{2} .
$$

Braneworld effects are still negligible.

We conclude that in traditional lensing scenarios with black holes that are stellar mass or larger, the lensing scale is simply much too large to provide an effective probe of braneworld effects on the scale $\ell \lesssim 0.2 \mathrm{~mm}$.

\section{ATTOLENSING BY PRIMORDIAL BRANEWORLD BLACK HOLES}

To obtain larger braneworld effects, we need to consider even smaller black hole masses. In the braneworld 
model, black holes may be created in the early universe and survive to the present day with masses as small as $\sim 1 \mathrm{~kg} \sim 10^{-30} M_{\odot}$ [15, 16]. Primordial braneworld black holes could be detectable via lensing in the wave optics limit, in a phenomenon we call attolensing.

Attolensing is descended from the concept of femtolensing in general relativity, introduced by Gould [35]. A black hole of mass $M_{\bullet} \sim 10^{-16} M_{\odot}$ placed at a cosmological distance creates images with an angular spacing of order a femto-arcsecond. Femtolensing could produce observable interference fringes in the energy spectrum of a gamma-ray burst at energies in the range of $\mathrm{keV}$ to $\mathrm{MeV}$. Various aspects of the interference patterns produced by femtolensing have been studied for general relativity [36, 37, 38, 39].

Likewise, attolensing by primordial braneworld black holes would be observable through wave optics effects. There are, however, some notable differences between femtolensing in general relativity and attolensing in braneworld gravity. First, at a given black hole mass, braneworld gravity and general relativity predict slightly different interference patterns (see Section V). Second, braneworld gravity allows lower mass black holes to survive to the present day (compared with GR); as a result, there are energy scales at which interference effects would be observable for braneworld gravity but not general relativity. Third, as a point of terminology, Gould's term referred to the scale of the angular image separation. By contrast, we choose a term that denotes the mass scale, which for wave optics is much more fundamental than the angular image separation. As noted above, primordial braneworld black holes can have an enormous range of masses, down to $\sim 10^{-30} M_{\odot}$. In quantitative examples we take $M \sim 10^{-18} M_{\odot}$ to be illustrative (hence "attolensing"), but always quote the mass scaling.

The gravitational radius and time scale for attolensing are

$$
\begin{aligned}
& \mathrm{m}_{\bullet}=1.5 \times 10^{-13} \times\left(\frac{M_{\bullet}}{10^{-18} M_{\odot}}\right) \mathrm{cm} \\
& \tau_{E}=2.0 \times 10^{-23} \times\left(1+z_{L}\right)\left(\frac{M_{\bullet}}{10^{-18} M_{\odot}}\right) \mathrm{s}
\end{aligned}
$$

where to be general we consider that the lens may have cosmological redshift $z_{L}$. The bright interference fringes appear at energies (see eq. [59)

$$
\begin{aligned}
E_{j}^{\mathrm{br}=} & 210 \times \frac{j+1 / 4}{1+z_{L}} \frac{1}{\Lambda_{0}+\Lambda_{1}}\left(\frac{M_{\bullet}}{10^{-18} M_{\odot}}\right)^{-1} \times \\
& {\left[1-\frac{\Lambda_{0}}{\Lambda_{0}+\Lambda_{1}} \varepsilon_{\ell}^{2}+\mathcal{O}\left(\varepsilon_{\ell}\right)^{3}\right] \mathrm{MeV} }
\end{aligned}
$$

The dark fringes are found by replacing $j+1 / 4$ with $j+3 / 4$ (cf. eq. 61). The dimensionless factors involving $\Lambda_{0}$ and $\Lambda_{1}$ depend on the source position as shown in Figure 2 Braneworld effects introduce a fractional shift in the fringe energies (relative to GR) that is given by $\varepsilon_{\ell}^{2}$ times a factor that is approximately 0.5 for all relevant source positions.

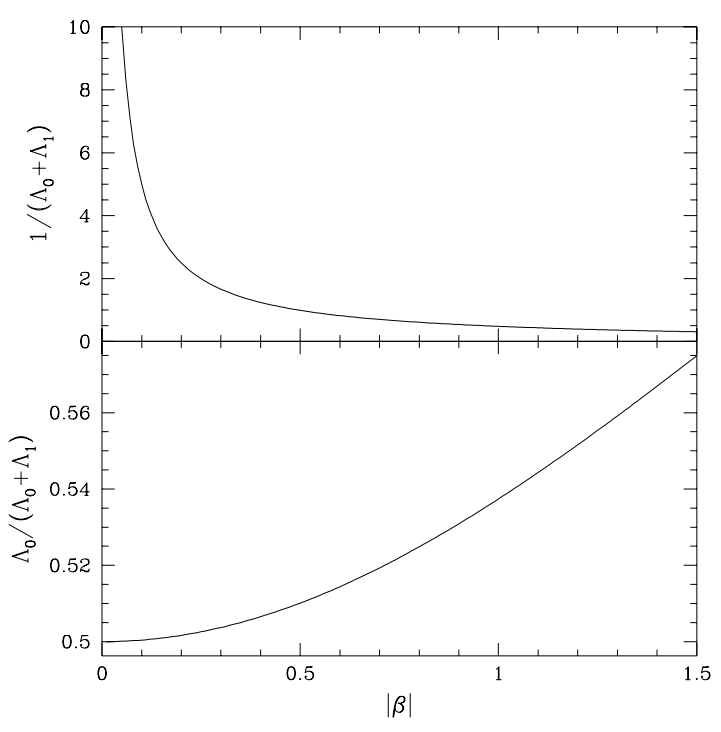

FIG. 2: Dimensionless factors in the energies of attolensing interference fringes (see eq. 71), which depend on the scaled source position $\beta$.

\section{A. Braneworld Black Holes in the Solar System}

Since the amplitude of braneworld effects decreases with the distance to the lens (see eq.65), we can estimate the maximum realistic effects by considering primordial braneworld black holes that are as close as possible to Earth. To estimate how close this might be, let $\rho_{\text {bh }}$ be the mean mass density in primordial braneworld black holes in the Solar neighborhood. We might take this to be some fraction $f_{\mathrm{bh}}$ of the density $\rho_{\mathrm{dm}}$ of dark matter in our region of the Galaxy. If all braneworld black holes have the same mass $M_{\bullet}$, then their number density is $n_{\mathrm{bh}}=\rho_{\mathrm{bh}} / M_{\bullet}$, so the typical distance between black holes is

$$
d \sim n_{\mathrm{bh}}^{-1 / 3}=\left(\frac{f_{\mathrm{bh}} \rho_{\mathrm{dm}}}{M_{\bullet}}\right)^{-1 / 3} .
$$

Detailed modeling of our Galaxy indicate that the density of dark matter in the Solar neighborhood is $\rho_{\mathrm{dm}}=$ $(0.011 \pm 0.005) M_{\odot} \mathrm{pc}^{-3}[40]$. This dark matter density yields

$$
d_{L} \sim 0.93 \times f_{\mathrm{bh}}^{-1 / 3}\left(\frac{M_{\bullet}}{10^{-18} M_{\odot}}\right)^{1 / 3} \mathrm{AU} .
$$

Note that the natural unit here is Astronomical Units. In other words, if $M_{\bullet} / M_{\odot} \lesssim 10^{-13} f_{\mathrm{bh}}$ then the nearest primordial black holes reside within our Solar System!

Put another way, a simple estimate of the total mass in primordial black holes within the Solar System is

$$
M_{\mathrm{bh}} \sim \frac{4}{3} \pi R_{P}^{3} \rho_{\mathrm{bh}} \sim 3.3 \times 10^{-13} f_{\mathrm{bh}} M_{\odot},
$$


where $R_{P} \approx 40 \mathrm{AU}$ is the radius of Pluto's orbit. This corresponds to a total number

$$
N_{\mathrm{bh}} \sim 3.3 \times 10^{5} \times f_{\mathrm{bh}}\left(\frac{M}{10^{-18} M_{\odot}}\right)^{-1}
$$

of primordial braneworld black holes in the volume interior to Pluto's orbit.

The angular Einstein radius and braneworld correction scale for a primordial braneworld black hole in the Solar System are

$$
\begin{aligned}
\vartheta_{E} \sim & 4.1 \times 10^{-8} \times \\
& \left(\frac{M_{\bullet}}{10^{-18} M_{\odot}}\right)^{1 / 2}\left(\frac{d_{L}}{\mathrm{AU}}\right)^{-1 / 2} \operatorname{arcsec} \\
\varepsilon_{\ell}^{2} \sim & 4.5 \times 10^{-5} \times \\
& \left(\frac{\ell}{0.2 \mathrm{~mm}}\right)^{2}\left(\frac{M_{\bullet}}{10^{-18} M_{\odot}}\right)^{-1}\left(\frac{d_{L}}{\mathrm{AU}}\right)^{-1} .
\end{aligned}
$$

In other words, braneworld corrections for lensing by primordial braneworld black holes in the Solar System are small but not absurdly so.

The primordial braneworld black holes we are considering are much less massive than asteroids. In fact, the total mass in (74) is smaller than many large asteroids [41], and is well within upper limits on dark matter in the Solar System derived from Solar System dynamics [42, 43. . Our estimate of the mass and number of primordial black holes in the Solar System is based on the assumption that the dark matter is distributed uniformly in the Solar neighborhood, which seems plausible because stars and the star formation process are inefficient at capturing dark matter 42 .

\section{B. Braneworld Black Holes in the Cosmos}

Rather than considering braneworld black holes in our Solar System, we may imagine them spread throughout the universe. The angular Einstein radius and braneworld correction scale for a primordial braneworld black hole at a cosmological distance are

$$
\begin{aligned}
\vartheta_{E} \sim 2.9 \times 10^{-15} \times & \left(\frac{M_{\bullet}}{10^{-18} M_{\odot}}\right)^{1 / 2}\left(\frac{d_{L S}}{d_{L} d_{S}}\right)^{1 / 2} \operatorname{arcsec}, \\
\varepsilon_{\ell}^{2} \sim 2.2 \times 10^{-19} \times & \left(\frac{\ell}{0.2 \mathrm{~mm}}\right)^{2}\left(\frac{M_{\bullet}}{10^{-18} M_{\odot}}\right)^{-1}\left(\frac{d_{S}}{d_{L} d_{L S}}\right),
\end{aligned}
$$

where the distances are all in Gpc. In other words, both the angular image separation and the braneworld corrections would be difficult to measure for cosmological primordial braneworld black holes. However, the interference fringes are still very similar to the Solar System case; they shift only by the order unity factor $1+z_{L}$ (see eq. (71).
An obvious question is whether the probability of cosmological attolensing is high enough to be interesting. Suppose that primordial braneworld black holes con-

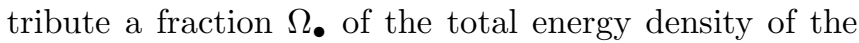
universe. The lensing optical depth - defined to be the fraction of the sky covered by Einstein radii, which is useful not only for traditional lensing but for attolensing as well - would then be $\tau$ such that $\tau / \Omega$ • $\gtrsim 0.1$, with the precise coefficient determined by the distribution of source redshifts 35, 44]. The optical depth is independent of the distribution of primordial black hole masses, and is nearly equal to the attolensing probability [45]. In other words, if primordial black holes contribute a cosmologically significant fraction of matter, then the attolensing probability is not very small.

\section{On Observing Attolensing}

For our example black hole of mass $M_{\bullet}=10^{-18} M_{\odot}$, eq. (71) indicates that semi-classical wave optics effects would be seen at energies of tens to hundreds of $\mathrm{MeV}$. This energy range will soon be accessible with the GLAST satellite, scheduled for launch in 2007 [46]. (At present, energies up to $8 \mathrm{MeV}$ can be observed with the INTEGRAL satellite [47]. There may be important attolensing effects that could be seen at these lower energies, but they would involve physical optics effects and it is not yet possible to examine braneworld black hole lensing in the physical optics regime [see Section $\nabla$.)

It is instructive to assess whether GLAST could detect the fringe pattern in an attolensed gamma-ray burst. At high energies, a typical gamma-ray burst has a power law spectrum such that the number of photons per unit energy is

$$
\frac{d N_{0}}{d E} \propto E^{-\eta},
$$

with $\eta \approx 2.25$ [48]. If the gamma-ray burst is attolensed, its observed energy spectrum equals the intrinsic spectrum multiplied by the energy-dependent lensing magnification $\mathcal{M}$ from eq. (57),

$$
\frac{d N_{\mathrm{obs}}}{d E} \propto \mathcal{M}(E) E^{-\eta} .
$$

Figure 3 shows a sample energy spectrum for a gammaray burst attolensed by a braneworld black hole with mass $M_{\bullet}=10^{-18} M_{\odot}$, for a configuration in which the scaled source position is $\beta=0.75$. The dark fringes (valleys) appear to be more prominent than the bright fringes (peaks), although this is an artifact of using a logarithmic vertical scale. Figure 3 b then shows an example of a binned spectrum as it might be observed by GLAST. In this example, the bin width is about three times GLAST's energy resolution, and the errorbars indicate the statistical uncertainties if there are 100 photons detected with energies between $30 \mathrm{MeV}$ and $1 \mathrm{GeV}$. 


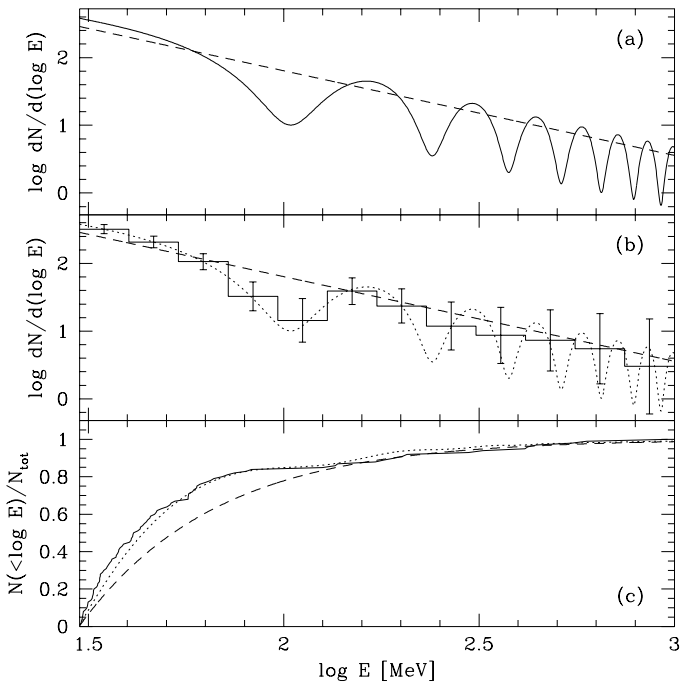

FIG. 3: Sample energy spectrum of a gamma-ray burst attolensed by a braneworld black hole with mass $M_{\bullet}=$ $10^{-18} M_{\odot}$, for a configuration in which the scaled source position is $\beta=0.75$. (a) The dashed lines shows the intrinsic energy spectrum, while the dotted line shows the attolensed spectrum (both normalized to 100 photons). We follow the convention for high-energy spectra and plot the logarithm of the number of photons per logarithmic energy interval. (b) The dashed and dotted lines reproduce the ideal spectra from panel (a). The histogram shows a sample "observation" as it might be seen by GLAST. The errorbars indicate the uncertainty in the "observed" spectrum if the telescope records 100 photons with energies between $30 \mathrm{MeV}$ and $1 \mathrm{GeV}$. (c) Cumulative distribution of photon energies. The dashed line shows the distribution without lensing; the dotted line shows the predicted distribution with lensing; while the solid line shows the distribution for a mock observation of 100 photons. A Kolmogorov-Smirnov test rejects the hypothesis that this gamma-ray burst was not lensed at more than $99 \%$ confidence.

The "observed" spectrum shows an excess near 30-40 $\mathrm{MeV}$, and a clear dip near $100 \mathrm{MeV}$, relative to the unlensed spectrum. To determine the significance of such features, and more generally to assess the ability to distinguish between the unlensed and attolensed cases, we use the Kolmogorov-Smirnov test to compare the "observed" spectrum to the unlensed power law spectrum. (The KS test applies to the cumulative distribution of photon energies, shown in Figure 3r.) For this combination of mass and source position, 100 counts is typically sufficient to reject the hypothesis that the gamma-ray burst was not lensed at more than $99 \%$ confidence. While the number of counts required to reach $99 \%$ confidence varies with the black hole mass, and to a lesser extent the source position, it is important to see that for a typical scenario strong results could be obtained with as few as 100 counts. GLAST is expected to see of order 20 gamma-ray bursts per year with more than 100 counts above $30 \mathrm{MeV}$ [4]].

\section{On Identifying Primordial Braneworld Black Holes}

Explicit braneworld effects (characterized by $\varepsilon_{\ell}^{2}$ ) are vanishingly small for cosmological scenarios, although they might be measurable in Solar System scenarios. This does not limit our ability to test braneworld gravity, though. The reason is that the braneworld model makes very different predictions, compared with general relativity, about the formation and survival of primordial black holes 13, 14, 15]. The starkest difference is that in GR all primordial black holes smaller than $\sim 10^{-19} M_{\odot}$ would have evaporated by the present day [12], while in braneworld gravity black holes as small as $\sim 1 \mathrm{~kg} \sim 10^{-30} M_{\odot}$ may be able to survive to today [15, 16]. Therefore, a clear detection of a black hole with mass $\lesssim 10^{-19} M_{\odot}$ would violate the GR prediction and support the braneworld model.

Observing attolensing fringes would not only reveal a primordial black hole but also place an important upper limit on its mass. From eq. (71), the energies of the bright and dark fringes are given by $\left(1+z_{L}\right)^{-1} M_{\bullet}^{-1}$ times a dimensionless factor of order unity that depends on the source position, $\beta$. The source position could be determined from the fringe amplitudes (see eq. 63). Thus, analyzing the fringes would fix the combination $\hat{M}_{\bullet} \equiv\left(1+z_{L}\right) M_{\bullet}$. While it may be difficult or impossible to determine $z_{L}$, we would know that $z_{L} \geq 0$ and hence $M_{\bullet} \leq \hat{M}_{\bullet}$. Since we would be seeking evidence that there are black holes below some mass threshold, upper limits available from attolensing would be useful and important.

\section{CONCLUSIONS}

We have presented a rigorous and comprehensive analytical formalism for gravitational lensing due to a braneworld black hole described by the Garriga-Tanaka metric. Using invariant quantities, we calculated all the fundamental geometric optics lensing observables. We then used these results to pursue a new direction in braneworld black hole lensing: wave optics. We computed the total magnification in the limit of semi-classical wave optics, and gave explicit formulas for the locations of the bright and dark interference fringes in the energy spectrum of a source lensed by a braneworld black hole.

Applying our results to realistic examples of lensing indicates that traditional lensing scenarios involving stellar-mass or supermassive black holes will be unable to test braneworld gravity in the foreseeable future. However, attolensing by primordial braneworld black holes does provide a powerful opportunity to probe braneworld effects via interference fringes in the energy spectra of gamma-ray bursts. If primordial braneworld black holes contribute a non-negligible fraction of the dark matter, there should be many within our Solar System. These nearby primordial black holes could be used 
to test braneworld gravity directly by looking for the $\varepsilon_{\ell}^{2}$ correction terms in the interference pattern, and indirectly by looking for primordial black holes smaller than the evaporation limit predicted for general relativity. Primordial black holes would also be spread throughout the cosmos. They may produce a measurable probability for attolensing of gamma-ray bursts, which would again afford the possibility of detecting black holes smaller than the GR limit.

It is worth reiterating that a population of primordial braneworld black holes in the Solar System is not ruled out by current dynamical constraints [42, 43]. In the future, the Laser Interferometry Space Antenna (LISA) may be able to detect gravitational impulses from passing primordial black holes, but LISA's sensitivity and noise from the Moon limit the detectability to $M_{\bullet} \gtrsim$ $10^{-19} M_{\odot}[50]$. Cosmologically, the lack of obvious femtolensing in a sample of 118 gamma-ray bursts places a weak upper limit on the cosmological density of primordial black holes in the mass range $10^{-16}-10^{-13} M_{\odot}[51]$; but the current constraint neither excludes fractions as high as $\Omega_{\bullet} \sim 0.1$ nor probes masses smaller than about $10^{-16} M_{\odot}$. In summary, current gravitational constraints do not rule out a substantial population of primordial black holes; for the future, attolensing may be the only way to probe the important mass scale below $10^{-19} M_{\odot}$.

While our analysis has been as rigorous and comprehensive as possible, there is one significant limitation. Since the full metric describing the spacetime around a braneworld black hole is still unknown, we cannot extend our analysis of lensing to the physical optics regime at the present time. We hope that this new fundamental test of braneworld gravity will motivate further attempts to determine the full metric for a braneworld black hole.

\section{Acknowledgments}

We thank Tom Banks, Ernesto Eiroa, Archan Majumdar, Takahiro Tanaka, and Richard Whisker for helpful correspondences on braneworld gravity. We thank the anonymous referee for valuable suggestions. This work was supported in part by NSF grants DMS-0302812, AST-0434277, and AST-0433809.
[1] P. Schneider, J. Ehlers, and E. E. Falco, Gravitational Lenses (Berlin: Springer, 1992).

[2] A. O. Petters, H. Levine, and J. Wambsganss, Singularity Theory and Gravitational Lensing (Boston: Birkhauser, 2001).

[3] C. S. Kochanek, P. Schneider, and J. Wambsganss, Gravitational Lensing: Strong, Weak, and Micro. Lecture Notes of the 33rd Saas-Fee Advanced Course, ed. G. Meylan, P. Jetzer, and P. North (Berlin: Springer-Verlag).

[4] C. R. Keeton and A. O. Petters, Phys. Rev. D 72, 104006 (2005).

[5] C. R. Keeton and A. O. Petters, Phys. Rev. D 73, 044024 (2006).

[6] L. Randall and R. Sundrum, Phys. Rev. Lett. 83, 4690 (1999).

[7] C. D. Hoyle, U. Schmidt, B. R. Heckel, E. G. Adelberger, J. H. Gundlach, D. J. Kapner, and H. E. Swanson, Phys. Rev. Lett. 86, 1418 (2001); J. Long, H. Chan, A. Churnside, E. Gulbis, M. Varney, and J. Price, Nature 421, 922 (2003).

[8] T. Banks and W. Fischler, hep-th/9906038 (1999); R. Emparan, G. T. Horowitz, and R. C. Myers, Phys. Rev. Lett. 85, 499 (2000); S. Dimopoulos and G. Landsberg, Phys. Rev. Lett. 87, 161602 (2001); L. Anchordoqui and H. Goldberg, Phys. Rev. D 67, 064010 (2003); M. Cavaglia, S. Das, and R. Maartens, Class. Quant. Grav. 20, L205 (2003); A. Chamblin, F. Cooper, and G. C. Nayak, Phys. Rev. D 69, 065010 (2004).

[9] J. L. Feng and A. D. Shapere, Phys. Rev. Lett. 88, 021303 (2002); L. Anchordoqui and H. Goldberg, Phys. Rev. D 65, 047502 (2002); E. J. Ahn, M. Ave, M. Cavaglia, and A. V. Olinto, Phys. Rev. D 68, 043004 (2003).

[10] S. W. Hawking, Mon. Not. R. Astron. Soc. 152, 75
(1971); B. J. Carr and S. W. Hawking, Mon. Not. R. Astron. Soc. 168, 399 (1974); B. J. Carr, Astrophys. J. 201, 1 (1975).

[11] Y. Sendouda, S. Nagataki, and K. Sato, astro-ph/0603509 (2006).

[12] D. N. Page, Phys. Rev. D 13, 198 (1976); D. N. Page, Phys. Rev. D 14, 3260 (1976).

[13] R. Guedens, D. Clancy, and A. R. Liddle, Phys. Rev. D 66, 043513 (2002).

[14] R. Guedens, D. Clancy, and A. R. Liddle, Phys. Rev. D 66, 083509 (2002).

[15] A. S. Majumdar, Phys. Rev. Lett. 90, 031303 (2003).

[16] A. S. Majumdar and N. Mukherjee, Intl. Jnl. Mod. Phys. D 14, 1095 (2005).

[17] J. Garriga and T. Tanaka, Phys. Rev. Lett. 84, 2778 (2000).

[18] S. Giddings, E. Katz, and L. Randall, JHEP 0003, 023 (2000).

[19] M. Sasaki, T. Shiromizu, and K. I. Maeda, Phys. Rev. D 62, 024008 (2000).

[20] R. Gregory, R. Whisker, K. Beckwith, and C. Done, J. Cosmology and Particle Physics, 10 (013), 1 (2004).

[21] S. Kar and M. Sinha, Gen. Relativ. Gravit. 35, 1775 (2003).

[22] A. S. Majumdar and N. Mukherjee, Mod. Phys. Lett. A 20, 2487 (2005).

[23] E. F. Eiroa, Phys. Rev. D 71, 083010 (2005).

[24] R. Whisker, Phys. Rev. D 71, 064004 (2005).

[25] K. S. Virbhadra, D. Narasimha, and S. M. Chitre, Astron. Astrophys. 337, 1 (1998); K. S. Virbhadra and G. F. R. Ellis, Phys. Rev. D 62, 084003 (2002).

[26] A. O. Petters, Mon. Not. R. Astron. Soc. 338, 457 (2003).

[27] T. Nakamura and S. Deguchi, Prog. Theor. Physics. Suppl., 133, 137 (1999). 
[28] R. Bean and J. Magueijo, Phys. Rev. D. 66, 063505 (2002).

[29] A. M. Ghez, S. Salim, S. D. Hornstein, A. Tanner, J. R. Lu, M. Morris, E. E. Becklin, and G. Duchêne, Astrophys. J. 620, 744 (2005).

[30] F. Eisenhauer, R. Schödel, R. Genzel, T. Ott, M. Tecza, R. Abuter, A. Eckart, and T. Alexander, Astrophys. J. 597, L121 (2003).

[31] B. Pacyński, Ann. Rev. Astron. Astrophys. 34, 419 (1996)

[32] S. Gaudi and A. O. Petters, Astrophys. J. 574, 970 (2002); S. Gaudi and A. O. Petters, Astrophys. J. 580, 468 (2002).

[33] M. Burgay et al., Nature 426, 531 (2003); G. Lyne et al., Science 303, 1153 (2004).

[34] D. Lai and R. R. Rafikov, Astrophys. J. 621, L41 (2005); R. R. Rafikov and D. Lai, astro-ph/0503461 (2005); R. R. Rafikov and D. Lai, astro-ph/0512417 (2005).

[35] A. Gould, Astrophys. J. 386, L5 (1992).

[36] K. Z. Stanek, B. Paczyński, and J. Goodman, Astrophys. J, 413, L7 (1993).

[37] M. Jarosyński and B. Paczyński, Astrophys. J. 455, 443 (1995).

[38] A. Ulmer and J. Goodman, Astrophys. J. 442, 67 (1995).
[39] A. Gould and B. S. Gaudi, Astrophys. J. 486, 687 (1997).

[40] R. P. Olling and M. R. Merrifield, Mon. Not. R. Astron. Soc. 326, 164 (2001).

[41] J. L. Hilton, Astron. J. 117, 1077 (1999).

[42] J. D. Anderson et al., Astrophys. J. 342, 539 (1989); J. D. Anderson et al., Astrophys. J. 448, 885 (1995).

[43] Ø. Grøn and H. H. Soleng, Astrophys. J. 456, 445 (1996).

[44] W. H. Press and J. E. Gunn, Astrophys. J. 185, 397 (1973); E. L. Turner, J. P. Ostriker, and J. R. Gott, Astrophys. J. 284, 1 (1984).

[45] D. E. Holz and R. M. Wald, Phys. Rev. D 58, 063501 (1998).

[46] http://glast.gsfc.nasa.gov

[47] http://isdc.unige.ch

[48] R. D. Preece et al., Astrophys. J. 496, 849 (1998); R. D. Preece et al., Astrophys. J. Supp. 126, 19 (2000).

[49] N. Omodei et al., astro-ph/0603762 (2006).

[50] N. Seto \& A. Cooray, Phys. Rev. D 70, 063512 (2004). A. W. Adams \& J. S. Bloom, astro-ph/0405266 (2004).

[51] G. F. Marani et al., Astrophys. J. Lett. 512, 13 (1999).

[52] Asymptotic flatness can be generalized to a RobertsonWalker background by using angular diameter distances and including appropriate redshift factors; see Section 